\title{
The Fourth Fundamental Form of the Torus Hypersurface
}

\author{
Erhan Güler \\ Department of Mathematics, Faculty of Sciences, Bartın University, 74100 Bartın, Turkey \\ e-mail: eguler@bartin.edu.tr
}

\begin{abstract}
We introduce the fourth fundamental form of the torus hypersurface in the four dimensional Euclidean space. We also compute I, II, III and IV fundamental forms of a torus hypersurface.
\end{abstract}

\section{Introduction}

Surfaces and hypersurfaces have been worked by the mathematicians for centuries. We see some new papers about torus surfaces and torus hypersurfaces in the literature such as [2-15].

Aminov [1] gave the three dimensional submanifold $M^{3}$ in $\mathbb{E}^{4}$, homeomorphic to $S^{1} \times S^{2}$, considering in a similar way to the construction of an ordinary torus in $\mathbb{E}^{3}$.

Let $\gamma$ be a circle of radius $R$ with the center at the origin $O$ in a coordinate plane $\mathbb{E}^{2}$, and $P$ be a point of $\gamma$. Spanning $\mathbb{E}^{3}$ on vectors $O P, e_{3}, e_{4}$, we consider the sphere $S^{2}(P)$ of radius $r$ with the center at $P$. When $P$ moves along $\gamma$, then all points of $S^{2}(P)$ form the submanifold $M^{3}$ in $\mathbb{E}^{4}$, and then a torus hypersurface in $\mathbb{E}^{4}$ can be parametrized by:

$$
\mathbf{x}(u, v, w)=\left(\begin{array}{c}
(R+r \cos u \cos v) \cos w \\
(R+r \cos u \cos v) \sin w \\
r \cos u \sin v \\
r \sin u
\end{array}\right)
$$

where $u, v, w \in I \subset \mathbb{R}$.

In this paper, we study the fourth fundamental form of the torus hypersurface in the four dimensional Euclidean space $\mathbb{E}^{4}$. We present fundamental notions of four

Received: October 19, 2020; Accepted: October 26, 2020

2010 Mathematics Subject Classification: 53A35, 53C42.

Keywords and phrases: four space, torus hypersurface, fourth fundamental form. 
dimensional Euclidean geometry. Moreover, we give fundamental forms I, II, III, and IV of torus hypersurface.

\section{Preliminaries}

We consider characteristic polynomial of shape operator $\mathbf{S}$ :

$$
P_{\mathbf{S}}(\lambda)=0=\operatorname{det}\left(\mathbf{S}-\lambda I_{n}\right)=\sum_{k=0}^{n}(-1)^{k} s_{k} \lambda^{n-k},
$$

where $I_{n}$ denotes the identity matrix of order $n$ in $\mathbb{E}^{n+1}$. Then, we get curvature formulas

$$
\left(\begin{array}{c}
n \\
i
\end{array}\right) \mathfrak{c}_{i}=s_{i}
$$

Here, $\left(\begin{array}{l}n \\ 0\end{array}\right) \mathfrak{c}_{0}=s_{0}=1$ by definition. So, $k$-th fundamental form of hypersurface $M^{n}$ is defined by

$$
\mathrm{I}\left(\mathbf{S}^{k-1}(X), Y\right)=\left\langle\mathbf{S}^{k-1}(X), Y\right\rangle .
$$

Then, we get

$$
\sum_{i=0}^{n}(-1)^{i}\left(\begin{array}{c}
n \\
i
\end{array}\right) \mathfrak{c}_{i} \mathrm{I}\left(\mathbf{S}^{k-1}(X), Y\right)=0
$$

In the rest of this paper, we shall identify a vector $(a, b, c, d)$ with its transpose $(\mathrm{a}, \mathrm{b}, \mathrm{c}, \mathrm{d})^{t}$.

Let $\mathbf{M}=\mathbf{M}(u, v, w)$ be an isometric immersion of a hypersurface $M^{3}$ in $\mathbb{E}^{4}$. Inner product of vectors $\vec{x}=\left(x_{1}, x_{2}, x_{3}, x_{4}\right)$ and $\vec{y}=\left(y_{1}, y_{2}, y_{3}, y_{4}\right)$ in $\mathbb{E}^{4}$ is given by as follows:

$$
\langle\vec{x}, \vec{y}\rangle=x_{1} y_{1}+x_{2} y_{2}+x_{3} y_{3}+x_{4} y_{4}
$$

Vector product $\vec{x} \times \vec{y} \times \vec{z} \quad$ of $\vec{x}=\left(x_{1}, x_{2}, x_{3}, x_{4}\right), \quad \vec{y}=\left(y_{1}, y_{2}, y_{3}, y_{4}\right), \quad \vec{z}=$ $\left(z_{1}, z_{2}, z_{3}, z_{4}\right)$ in $\mathbb{E}^{4}$ is defined by as follows:

$$
\vec{x} \times \vec{y} \times \vec{z}=\operatorname{det}\left(\begin{array}{l}
e_{1} e_{2} e_{3} e_{4} \\
x_{1} x_{2} x_{3} x_{4} \\
y_{1} y_{2} y_{3} y_{4} \\
z_{1} z_{2} z_{3} z_{4}
\end{array}\right) .
$$

The Gauss map of a hypersurface $\mathbf{M}$ is given by 


$$
e=\frac{\mathbf{M}_{u} \times \mathbf{M}_{v} \times \mathbf{M}_{w}}{\left\|\mathbf{M}_{u} \times \mathbf{M}_{v} \times \mathbf{M}_{w}\right\|^{\prime}}
$$

where $\mathbf{M}_{u}=d \mathbf{M} / d u$. For a hypersurface $\mathbf{M}$ in $\mathbb{E}^{4}$, we have following fundamental form matrices

$$
\begin{gathered}
\mathrm{I}=\left(\begin{array}{lll}
E & F & A \\
F & G & B \\
A & B & C
\end{array}\right), \\
\mathrm{II}=\operatorname{det}\left(\begin{array}{lll}
L & M & P \\
M & N & T \\
P & T & V
\end{array}\right), \\
\mathrm{III}=\left(\begin{array}{lll}
X & Y & O \\
Y & Z & R \\
O & R & S
\end{array}\right) .
\end{gathered}
$$

Here, the coefficients are given by

$$
\begin{aligned}
& E=\left\langle\mathbf{M}_{u}, \mathbf{M}_{u}\right\rangle, \quad F=\left\langle\mathbf{M}_{u}, \mathbf{M}_{v}\right\rangle, \quad G=\left\langle\mathbf{M}_{v}, \mathbf{M}_{v}\right\rangle, \quad A=\left\langle\mathbf{M}_{u}, \mathbf{M}_{w}\right\rangle, \quad B=\left\langle\mathbf{M}_{v}, \mathbf{M}_{w}\right\rangle, \\
& C=\left\langle\mathbf{M}_{w}, \mathbf{M}_{w}\right\rangle, \\
& L=\left\langle\mathbf{M}_{u u}, e\right\rangle, \quad M=\left\langle\mathbf{M}_{u v}, e\right\rangle, \quad N=\left\langle\mathbf{M}_{v v}, e\right\rangle, \quad P=\left\langle\mathbf{M}_{u w}, e\right\rangle, \quad T=\left\langle\mathbf{M}_{v w}, e\right\rangle, \\
& V=\left\langle\mathbf{M}_{w w}, e\right\rangle, \\
& X=\left\langle e_{u}, e_{u}\right\rangle, \quad Y=\left\langle e_{u}, e_{v}\right\rangle, \quad Z=\left\langle e_{v}, e_{v}\right\rangle, \quad O=\left\langle e_{u}, e_{w}\right\rangle, \quad R=\left\langle e_{v}, e_{w}\right\rangle, \\
& S=\left\langle e_{w}, e_{w}\right\rangle,
\end{aligned}
$$

and $e$ is the Gauss map (i.e. the unit normal vector field).

\section{The Fourth Fundamental Form}

Next, we obtain the fourth fundamental form matrix for a hypersurface $\mathbf{M}(u, v, w)$ in $\mathbb{E}^{4}$. Using characteristic polynomial $P_{\mathbf{S}}(\lambda)=a \lambda^{3}+b \lambda^{2}+c \lambda+d=0$, we obtain curvature formulas: $\mathfrak{c}_{0}=1$ (by definition),

$$
\mathfrak{c}_{1}=-\frac{b}{\left(\begin{array}{l}
3 \\
1
\end{array}\right) a}, \quad \mathfrak{c}_{2}=\frac{c}{\left(\begin{array}{l}
3 \\
2
\end{array}\right) a}, \quad \mathfrak{c}_{3}=-\frac{d}{\left(\begin{array}{l}
3 \\
3
\end{array}\right) a} .
$$

Theorem 3.1. For any hypersurface $M^{3}$ in $\mathbb{E}^{4}$, the fourth fundamental form is related by

$$
\mathfrak{c}_{0} \mathrm{IV}-3 \mathfrak{C}_{1} \mathrm{III}+3 \mathfrak{c}_{2} \mathrm{II}-\mathfrak{C}_{3} \mathrm{I}=0 .
$$


Proof. Taking $n=3$ in (2.2), then some computing, we get the fourth fundamental form matrix as follows

$$
\mathrm{IV}=\left(\begin{array}{lll}
\zeta & \eta & \delta \\
\eta & \phi & \sigma \\
\delta & \sigma & \xi
\end{array}\right)
$$

where

$$
\zeta=-\frac{\left\{\begin{array}{c}
C L^{2} N-C L M^{2}-G L P^{2}+B^{2} L X+A^{2} N X+G L^{2} V+F^{2} V X+N P^{2} E+M^{2} V E \\
-C N X E-G V X E-C G L X+2\left(B T X E-B L^{2} T-M P T E+A B M X-A L N P\right. \\
+B L M P+A L M T+C F M X+A G P X-B F P X-A F T X-F L M V+F L P T)
\end{array}\right\}}{\operatorname{det} \mathrm{I}}
$$

$\eta=\left\{\begin{array}{c}C M^{3}-F N P^{2}-G M P^{2}-F L T^{2}-B^{2} L Y-A^{2} N Y+F M^{2} V-F^{2} V Y+M T^{2} E \\ +C N Y E-M N V E+G V Y E-C L M N+C G L Y+F L N V-G L M V+2(A F P Y \\ \left.-B T Y E+A B M Y+A N M P-B L M T-C F M Y-A G P Y+B F P Y-T M^{2} A-B M^{2} P\right)\end{array}\right.$

$\delta=\frac{\left\{\begin{array}{c}G P^{3}-B^{2} L O-A^{2} N O+A N P^{2}+C M^{2} P-A L T^{2}-A V M^{2}-F^{2} O V+P T^{2} E \\ +C N O E+G O V E-N P V E+C G L O-C L N P+A L N V-G L P V+2(A B M O \\ \left.-B O T E-C F M O-A G O P+B F O P+A F O T-B L P T+F M P V-B M P^{2}-F P^{2} T\right)\end{array}\right\}}{\operatorname{det} \mathrm{I}}$

$\phi=-\frac{\left\{\begin{array}{c}C L N^{2}-C M^{2} N-G L T^{2}+B^{2} L Z+A^{2} N Z+G M^{2} V+F^{2} V Z-N T^{2} E+N^{2} V E \\ -C N Z E-G V Z E-C G L Z+2\left(-A N^{2} P+B T Z E-A B M Z+B M N P+A N M T\right. \\ -B L N T+C F M Z+A G P Z-B F P Z-A F T Z+F M N V+F N P T-G M P T)\end{array}\right\}}{\operatorname{det} \mathrm{I}}$,

$\sigma=\frac{\left\{\begin{array}{c}E T^{3}-B N P^{2}-B^{2} L R-A^{2} N R+B L T^{2}+C M^{2} T-B M^{2} V+G P^{2} T-F^{2} R V \\ +C N R E+G R V E-N T V E+C G L R-C L N T+B L N V-G L T V+2(A B M R \\ \left.-B R T E-C F M R-A G P R+B F P R+A F R T+A N P T+F M T V-A T^{2} M-F T^{2} P\right)\end{array}\right\}}{\operatorname{det} \mathrm{I}}$

$\xi=-\frac{\left\{\begin{array}{c}C N P^{2}-B^{2} L S-A^{2} N S+C L T^{2}+G L V^{2}-G P^{2} V+F^{2} S V+N V^{2} E-T^{2} V E \\ -C N S E-G S V E-C G L S+2\left(-F M V^{2}+B S T E-A B M S+C F M S+A G P S\right. \\ -B F P S-A F S T-C M P T-A N P V+B M P V+A T M V-B L T V-F P T V)\end{array}\right\}}{\operatorname{det} \mathrm{I}}$.

\section{Curvatures of Torus Hypersurface}

In this section, we compute curvatures of torus hypersurface (1.1).

With the first differentials of (1.1) depends on $u, v, w$, we get the Gauss map of (1.1): 


$$
e=-\left(\begin{array}{c}
\cos u \cos v \cos w \\
\cos u \cos v \sin w \\
\cos u \sin v \\
\sin u
\end{array}\right)
$$

We get the first and the second fundamental form matrices of (1.1), respectively,

$$
\begin{gathered}
\mathrm{I}=\left(\begin{array}{ccc}
r^{2} & 0 & 0 \\
0 & r^{2} \cos ^{2} u & 0 \\
0 & 0 & (R+r \cos u \cos v)^{2}
\end{array}\right), \\
\mathrm{II}=\left(\begin{array}{ccc}
r & 0 & 0 \\
0 & r \cos ^{2} u & 0 \\
0 & 0 & (R+r \cos u \cos v) \cos u \cos v
\end{array}\right) .
\end{gathered}
$$

Using $\mathrm{I}^{-1}$. II, torus hypersurface (1.1) in $\mathbb{E}^{4}$ has following shape operator

$$
\mathbf{S}=\left(\begin{array}{ccc}
k_{1} & 0 & 0 \\
0 & k_{2} & 0 \\
0 & 0 & k_{3}
\end{array}\right)=\left(\begin{array}{ccc}
\frac{1}{r} & 0 & 0 \\
0 & \frac{1}{r} & 0 \\
0 & 0 & \frac{\cos u \cos v}{R+r \cos u \cos v}
\end{array}\right)
$$

So, we compute the third fundamental form matrix using (4.1) of (1.1):

$$
\mathrm{III}=\left(\begin{array}{ccc}
1 & 0 & 0 \\
0 & \cos ^{2} u & 0 \\
0 & 0 & \cos ^{2} u \cos ^{2} v
\end{array}\right)
$$

Finally, using (3.2) on (1.1), we obtain the fourth quantities of (1.1), i.e., symmetric matrix, as follows

$$
\mathrm{IV}=\left(\begin{array}{ccc}
\frac{1}{r} & 0 & 0 \\
0 & \frac{\cos ^{2} u}{r} & 0 \\
0 & 0 & \frac{\cos ^{3} u \cos ^{3} v}{R+r \cos u \cos v}
\end{array}\right)
$$

Corollary 4.1. Torus hypersurface (1.1) in $\mathbb{E}^{4}$ has following relations

$$
\text { IV = III. S, }
$$




$$
\begin{gathered}
\text { III = II.S, } \\
\text { II = I.S. }
\end{gathered}
$$

Proof. Considering I, II, III, IV and $\mathbf{S}$ of (1.1), we obtain all quantities.

Corollary 4.2. Torus hypersurface (1.1) in $\mathbb{E}^{4}$ has following relations

$$
\frac{(\operatorname{detII})(\operatorname{detIII})^{2}}{(\operatorname{det})(\operatorname{detIV})^{2}}=\operatorname{det} \mathbf{S}=k_{1} k_{2} k_{3}=\frac{\cos u \cos v}{r^{2}(R+r \cos u \cos v)}=\mathfrak{c}_{3} .
$$

Proof. Using I, II, III, IV and $\mathbf{S}$ of (1.1), it is clear.

\section{Conclusion}

Torus hypersurfaces have been recently worked by a number of authors. We extend some well-known results of the torus hypersurfaces with the help of the fourth fundamental form

\section{References}

[1] Yu. Aminov, The Geometry of Submanifolds, Gordon and Breach Science Publishers, Amsterdam, 2001.

[2] V.A. Borovitskiı̌, K-closedness for weighted Hardy spaces on the torus $\mathrm{T}^{2}$, Zap. Nauchn. Sem. (POMI) 456 (2017), 25-36 (in Russian); translation in J. Math. Sci. (N.Y.) 234(3) (2018), 282-289. https://doi.org/10.1007/s10958-018-4004-9

[3] J. Dasgupta, B. Khan and V. Uma, Cohomology of torus manifold bundles, Math. Slovaca 69(3) (2019), 685-698. https://doi.org/10.1515/ms-2017-0257

[4] C.L. Duston, Torus solutions to the Weierstrass-Enneper representation of surfaces, $J$. Math. Phys. 60(8) (2019), 1-5. https://doi.org/10.1063/1.5097669

[5] J. Harvey and C. Searle, Almost non-negatively curved 4-manifolds with torus symmetry, Proc. Amer. Math. Soc. 148(11) (2020), 4933-4950. https://doi.org/10.1090/proc/15093

[6] M. Hasegawa and D. Ida, Instability of stationary closed strings winding around flat torus in five-dimensional Schwarzschild spacetimes, Phys. Rev. D 98(4) (2018), 1-7. https://doi.org/10.1103/PhysRevD.98.044045

[7] S. Hirose and E. Kin, On hyperbolic surface bundles over the circle as branched double covers of the 3-sphere, Proc. Amer. Math. Soc. 148(4) (2020), 1805-1814.

https://doi.org/10.1090/proc/14825 
[8] Y. Kamiyama, The orbit space of a hypersurface of a torus by an involution, JP J. Geom. Top. 21(4) (2018), 365-372. https://doi.org/10.17654/GT021040365

[9] E. Krasko and A. Omelchenko, Enumeration of $r$-regular maps on the torus. Part I: rooted maps on the torus, the projective plane and the Klein bottle, Sensed maps on the torus, Discrete Math. 342(2) (2019), 584-599. https://doi.org/10.1016/j.disc.2018.07.013

[10] E. Krasko and A. Omelchenko, Enumeration of $r$-regular maps on the torus. Part II: Unsensed maps, Discrete Math. 342(2) (2019), 600-614. https://doi.org/10.1016/j.disc.2018.09.004

[11] L.M. Lerman and K.N. Trifonov, The topology of symplectic partially hyperbolic automorphisms of the 4-torus, Mat. Zametki 108(3) (2020), 474-476 (in Russian). https://doi.org/10.1134/S0001434620090175

[12] M. Mase, Families of $K 3$ surfaces and curves of (2,3)-torus type, Kodai Math. J. 42(3) (2019), 409-430. https://doi.org/10.2996/kmj/1572487224

[13] S. Nakamura, The orthonormal Strichartz inequality on torus, Trans. Amer. Math. Soc. 373(2) (2020), 1455-1476. https://doi.org/10.1090/tran/7982

[14] Mauricio Poletti, Geometric growth for Anosov maps on the 3 torus, Bull. Braz. Math. Soc. (N.S.) 49(4) (2018), 699-713. https://doi.org/10.1007/s00574-018-0079-7

[15] T. Sakajo, Vortex crystals on the surface of a torus, Philos. Trans. Roy. Soc. A 377(2158) (2019), 1-17. https://doi.org/10.1098/rsta.2018.0344

This is an open access article distributed under the terms of the Creative Commons Attribution License (http://creativecommons.org/licenses/by/4.0/), which permits unrestricted, use, distribution and reproduction in any medium, or format for any purpose, even commercially provided the work is properly cited. 